\title{
4D scanning - The dynamic view on body measurements
}

\author{
Anke Klepser ${ }^{1, *}$, Simone Morlock ${ }^{1}$ \\ ${ }^{1}$ Hohenstein Institut fuer Textilinnovation g GmbH, Boennigheim, Germany \\ ${ }^{*}$ Corresponding author E-mail address: a.klepser@hohenstein.de
}

\section{INFO}

CDAPT, ISSN: 2701-939X

Peer reviewed article

2020, Vol.1, Nr. 1, pp.30-38

DOI:10.25367/cdatp.2020.1.p30-38

Received: 6 May 2020

Accepted: 7 June 2020

Available online: 22 September 2020

\begin{abstract}
Fitting test in movement are an important issue not only in work and sports wear since garment should not restrict the range of motion. Therefore, dynamic anthropometry is a major research topic. Until now, only static position could be captured. Still, it is not known how body geometry changes in dynamic movement. In IGF project "Mobilityrestrictions" photogrammetry scanner "Little Alice" was investigated regarding 4D. It enables serial recording in seconds. The aim of the research project was beside other to identify differences between static and dynamic body measurements.

Scanner "Little Alice" has never been utilized for body form analysis. Therefore, a basic research was performed. Several parameters were examined by iterative tests before scan procedure was defined. Three work or sport related movements were defined and compared to standard position: Biceps curl, leg flex and squat. The changes in scan surface were investigated by a three-step analysis: body measurements, cross sections and a 3D analysis. Scan procedure was performed by six test subjects German sizes 50 and 58 , age group $25-55$ years.

The results show that photogrammetry can be utilized to investigate body geometry changes due to movement. Body surface deviations have been investigated. Thus, not in all cases there were differences between static and dynamic scans. Yet, body geometry alters. 4D scanning enables comprehensive analysis of body geometry changes due to movement. Body measurement and surface alterations can be visualized and quantified. Scans of motions may be used to validate $3 D$ simulation avatars.
\end{abstract}

(C) 2020 The authors. Published by CDAPT.

This is an open access article under the CC BY-NC-ND license https://creativecommons.org/licenses/ peer-review under responsibility of the scientific committee of the CDAPT. 


\section{Introduction}

Dynamic anthropometry became one of the most important research topics in clothing technology in the last years [1,2]. To understand if and how the human body alters while it is moved is the foundation of ergonomic fit of personal protective equipment, work wear, sportswear and other functional garments [3]. In the past the assessment of body measurement alteration was only possible with measurement tape. This was time consuming and could only be realized with great effort [4]. With the development of 3D scanning systems an appropriate analysis was feasible [5]. Though, 3D body scanner technology enables only the capturing of static postures. Therefore, the scan shows the muscular system working to hold the position and not the interaction between agonist and antagonist in dynamic movement. It can be assumed that body proportion differs between dynamic and static status especially when performed by sporty subjects. The technological evolution from 3D to 4D scanning systems enables scanning in motion [6-8].

Garments are still developed on the basis of the anthropometric standard position defined in standards and sizing charts as: standing upright, legs hip wide spread, arms slightly abducted $[9,10]$. Although, the garment user is only seldom in exact this position. Mostly people are moving during the day. Especially in functional clothing like work or sportswear constant movement is performed. Therefore, it is of importance to analyze the body in motion, to extract significant measurement alterations and transfer the results into the garment development process. Kirk et al. (1966) were the first to develop a method to assess the enlargement and reduction of the body surface in connection with position changes. They applied measurement lines on the knee. Schmid et al. (1981) assumed that seam damages were caused by body geometry changes. Girth alterations at the waist-hip area in standing, sitting and squat posture were investigated by $\mathrm{Yu}$ et al. (2000). The research group of Ashdown analyzed dimensional changes on the basis of 3D data for the upper and the lower body half $[5,13,14,19]$. According to the state-of-the-art approach the modification of the body surface was investigated by static postures. Yet, so far there are just a few studies on measurement alteration due to movement $[9,10]$. Dynamic anthropometry was successfully applied mostly for the development of high performance sportswear. The goals were reduction of muscle fatigue, increase of physical comfort, reduction of resistance and performance increase [20,21]. Morlock et al. (2018) investigated for the first time body measurement differences between work and sports related positions over an entire size range of men and women [10].

So far, body movement is mostly captured with Motion Capture Technology. Defined landmarks on the body are tracked and transferred in digital data. Goals are e.g. video game design, computer animated movies or biomechanical analyzes in medicine and sports science. The output is an abstract skeleton for the assessment of range of motion. The acquisition of 3D surfaces with motion capturing has been investigated [22-26]. A large amount of markers are attached on the human body and tracked by the systems. In the beginning, 4D technology was utilized in medical research with computer tomography, magnetic resonance imaging and ultrasound. In the past years systems were developed to capture the body surface in a fast and precise way [6,7]. Mostly based on structural light and depth sensor technology. Where a defined pattern is projected on the object and captured by multiple cameras. Using algorithms and measurement principles e.g. triangulation or light section technique the surfaces are calculated. These high performance systems provide up to 180 frames per second in high resolution quality. Yet, they are very expansive and therefore mostly used by research institutes or big companies.

Photogrammetry seems to be a low cost alternative for small and medium enterprises to capture human bodies three- and four-dimensionally. Hereby, pictures are taken with digital reflex cameras from multiple angles. On the base of these picture sets a specific software calculates 3D models. The utilization of digital reflex cameras enables serial captures. Therefore, picture sets of the moving body can be taken. Each set represents one position of the movement. So far, three frames per second can be captured. If it is possible to take pictures of the most significant positions, most movements can be analyzed and body surface alterations identified. Until now, body measurements from scans can only be taken automatically from the so called a-pose. Taking measurements form the 3D-data of the moved body can only be realized interactive. From this point, the reduced data of photogrammetry systems is more of an advantage. Thus, photogrammetry full body scanners, so far not been utilized in this research field, should be investigated in regard of their operational capability in dynamic anthropometry focusing clothing technology 
applications. Like all 3D technologies they are only capable of the calculation of objects' surface that is visible to the sensors or cameras. Acquisition settings (area, light, camera set up) as well as the defined movement (reproducibility, feasibility) have a major impact on the visibility of the captured body. Capturing methods need to be developed with the aim to provide valid 3D data on which body surface changes due to movement can be examined.

\section{Method}

To investigate the operational capability in dynamic anthropometry focusing clothing technology applications 3D photogrammetry full body scanner "Little Alice" from 3Dcopysystems was utilized. The basic configuration is for three-dimensional scanning of human bodies. Objects are captured by 38 digital reflex cameras from multiple angles. Four computers are controlling the process, each one responsible for 9-10 cameras shooting synchronically. Three shots or frames per second can be performed. The pictures are transferred to a main computer on which photogrammetry software RealityCapture is utilized to calculate 3D-models. It is of importance that pictures of the captured object are overlapping. 38 cameras are the minimum amount to create full body scans. The overlapping areas enable automatic alignment of the pictures. The calculated 3D data consist of a solid mesh and texture information. Scanner "Little Alice" has never been utilized for body form analysis. Therefore, a basic research was performed. Six parameters were examined by iterative tests before scan procedure was defined: Shadowing of body areas, maximum of frames to be processed, acquisition area, movement speed, color and pattern of captured objects, data export and size accuracy. The aim of these tests was to generate 3D data with a minimum of shadowing and a maximum of accuracy on the one hand. On the other hand it was important to generate files that could be ease and fast imported in state of the art software of clothing technology research and development. These are programs to postprocess 3D data, to take automatically body measurements and 3D simulation applications.

As stated above, body measurements are undergoing changes while the body moves. To develop garments with optimal ergonomic fit the knowledge of the alterations is crucial. On the foundation of the basic research work in regard of the system's capabilities, three work or sport related movements were defined. The aim was to describe sequences that are typical for many working or sporting situations. In addition, the defined movements should lead to extreme changes in body proportions. The focus was the upper arm and thigh area. Reference to identify body surface alterations was the standard position (relaxed): standing upright, legs hip wide spread, arms slightly abducted. The defined body movements were: Biceps curl, leg flex and squat. All definitions are structured in a starting posture, a motion sequence and an ending posture. The scan procedure was performed by six test subjects in the German sizes 50 and 58 , age group $25-55$ years. Every participant wore tight fitting underwear. Anthropometric landmarks were highlighted by polystyrene balls of $2 \mathrm{~cm}$ diameter. The definition of the landmarks was performed according to Morlock et al. (2018) [10]. The following measurements were taken: body height, chest girth, waist girth, hip girth, across back, arm length, upper arm length, leg length, upper arm girth, thigh girth, knee girth and calf girth.

The changes in scan surface were investigated by a three-step analysis: body measurements, cross sections and a 3D analysis. Body measurements were taken from standard position and from the relevant position of de 4D scans. Software AnthroScan was utilized to take measurements automatically according to ISO 8559. To understand and quantify the difference in arm and leg length due to movement, the measurement definitions had to be adapted in those case. Arm length was taken from mid armfold to wrist. Leg length was taken from crotch height over knee to the floor. In a second step, cross sections of the upper arm and the thigh were taken in $1 \mathrm{~cm}$ distances. The assessment of the cross sections was performed in software Geomagic studio 2012 (curve generation) and Rhinoceros 6 (measurement). The value of each measurement position of upper arm and thigh at each scan was collected, respectively. Differences were identified and described. The last step were 3D analyzes utilizing Geomagic studio 2012. Scans of the standard position and the ending posture were postprocessed to generate smooth wraps. The full body scans were segmented and the right upper arm and the right thigh of the standard and the defined 4D posture were extracted. Then, segments were merged and a $3 D$ analysis describing the differences in geometry was performed. The results are so called heat maps that visualize the distance 
between the reference and the compared segment. With this method the interaction between agonist and antagonist (e.g. biceps and triceps) and the geometry alterations can be illustrated.

\section{Results}

The results of the basic research in regard of the operational capability showed that the scanner system "Little Alice" can be utilized for 3D and 4D scanning processes. Measurement and geometric differences between the scanned movement positions could be examined. In the first section of this chapter the results of the general assessment of the system are presented. In the second section the findings of the threestep analysis are shown.

With iterative tests the quality factors shadowing of body areas, maximum of frames to be processed, acquisition area, movement speed, color and pattern of captured objects, data export and size accuracy were assessed. As the 38 cameras stand for the minimum set up some quality reductions must be accepted. Areas between arms and torso or between legs at the crotch area demonstrate shadowing similar to other scanner systems. Yet, the multiple angels of the cameras reduce enable good capturing of most areas of the body. And the shadowing compared to full body scanners like e.g. laser scanners is significantly less in positions divergent to the standard posture. The vertical setting with the horizontal laser line of these systems lead to less data on horizontal body parts and obviously larger shadowing between limps and torso especially when arms or legs are positioned between column and torso.

The maximum amount of frames to take in a row was seven. Capturing more frame sets overstrained the computer system and pictures got mixed up on the transfer to the main computer. Therefore, movements have to be performed within three seconds. The acquisition area is determined by the systems structure. The base plate is ellipse shaped with a width of $230 \mathrm{~cm}$ and a depth of $168 \mathrm{~cm}$. The case is in an egg form with dimensions of $330 \times 320 \times 260 \mathrm{~cm}$ (length, width, height). The maximum area to capture objects measures $75 \times 75 \mathrm{~cm}$. The movement speed was no critical point for clothing technology applications. Fig. 1 shows jumping tests to investigate motion speed acceptance which underline the finding.

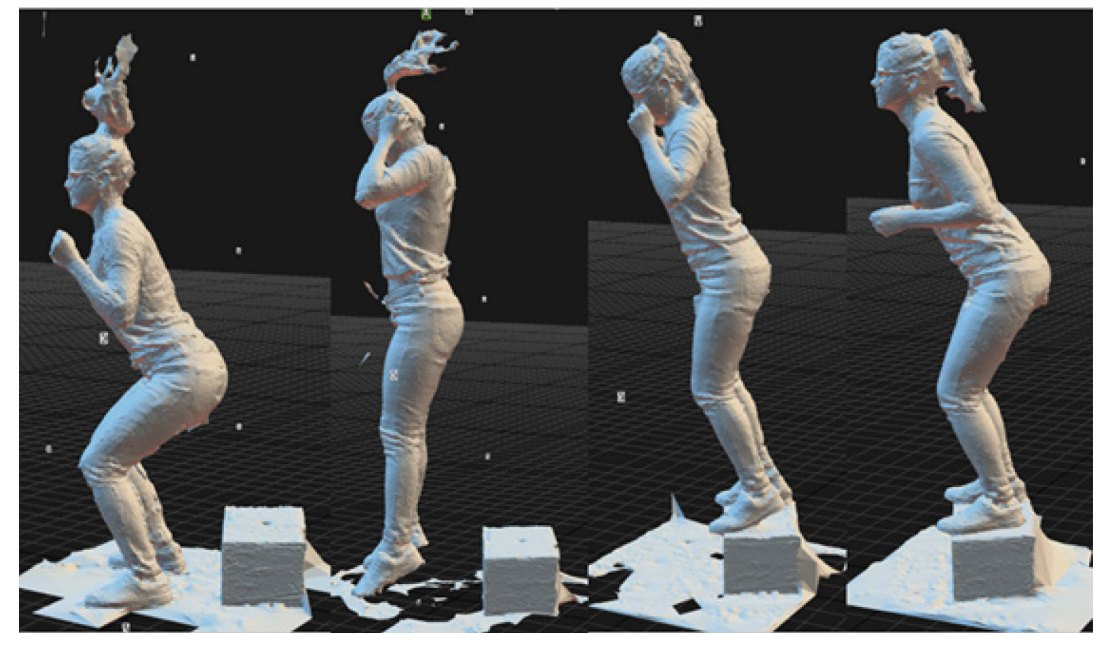

Fig. 1 Investigation motion speed acceptance.

To achieve optimal scan data quality the photogrammetry system can be supported by surfaces with pattern. Test showed that single-colored garment lead to decrease of geometry precision. Skin could be captured well, there was no difference in color. In a next step, the data export was examined. The mesh can be exported in different qualities in regard of resolution. Yet, quality affects file sizes and this has an impact on import and calculating times. Therefore, scans in three resolutions five million triangles, two million triangles and 500,000 triangles were exported in four different data formats (obj, ply, xyz and dxf). All were imported in standard utilized $3 D$ postprocessing and 3D simulation software (Antroscan, Geomagic Studio, Rhinocerus, CLO 3D and Browzwear). Import time, usability and visualization was assessed and rated from 1 to 4 , were 1 stands for very good and 4 for insufficient. It was shown, that objfiles provided the highest flexibility to be imported in other programs. In the lowest resolution $(500,000$ triangles) all the tested software were able to calculate and process the data. 
The last step of the basic investigation was the assessment of size accuracy. Since, the 3D / 4D data is generated to analyze body measurements and surface alterations this was the essential test. Three tailor dummies in the German sizes 50, 54 and 58 (bust girth 100, 108 and 116cm) were scanned in Vitus Smart XXL and with "Little Alice". All scans were measured in Anthroscan. The percentage variance was $1.5 \mathrm{~cm}$ in all primary measurements (bust girth, waist girth, hip girth and inseam length). This was rated as insufficient for the field of clothing technology. Therefore, the calibration process was optimized with the support of 3D copysystems. Afterwards, the percentage variance was $<0.3 \mathrm{~cm}$ in the defined measurements.

On the foundation of the basic research work three work or sport related movements were defined with a starting posture, sequence and ending posture (see Table 1).

Table 1. Defined body movements.

\begin{tabular}{|c|c|c|c|}
\hline Designation & Starting posture & Sequence & Ending posture \\
\hline Biceps curl & $\begin{array}{l}\text { Legs hip wide spread, } \\
\text { arms on shoulder level } \\
\text { stretch in front of the } \\
\text { body, hand to fists, back } \\
\text { of the hand to the floor, } \\
\text { extra load with } 1 \mathrm{~kg} \\
\text { dumbbells }\end{array}$ & $\begin{array}{l}\text { Elbow flexion up to } 90^{\circ} \\
\text { degree in elbow joint }\end{array}$ & $\begin{array}{l}\text { Legs hip wide spread, } \\
\text { arms on shoulder level, } \\
90^{\circ} \text { degree in elbow joint, } \\
\text { back of the hand to front }\end{array}$ \\
\hline Leg flex & $\begin{array}{l}\text { Legs hip wide spread, } \\
\text { arms in } 0^{\circ} \text { position }\end{array}$ & $\begin{array}{l}\text { Right leg lift up to hip and } \\
\text { knee flexion of } 90^{\circ} \\
\text { degrees, natural arm } \\
\text { movement, right foot is } \\
\text { positioned on platform, } \\
\text { left foot follows }\end{array}$ & $\begin{array}{l}\text { Legs hip wide spread, } \\
\text { arms in } 0^{\circ} \text { position }\end{array}$ \\
\hline squat & $\begin{array}{l}\text { Legs hip wide spread, } \\
\text { arms in } 0^{\circ} \text { position }\end{array}$ & $\begin{array}{l}\text { hip and knee flexion up to } \\
\text { of } 90^{\circ} \text { degrees, arms } \\
\text { moved stretched on the } \\
\text { side }\end{array}$ & $\begin{array}{l}\text { Squat with hip and knee } \\
\text { flexion up to of } 90^{\circ} \\
\text { degrees }\end{array}$ \\
\hline
\end{tabular}

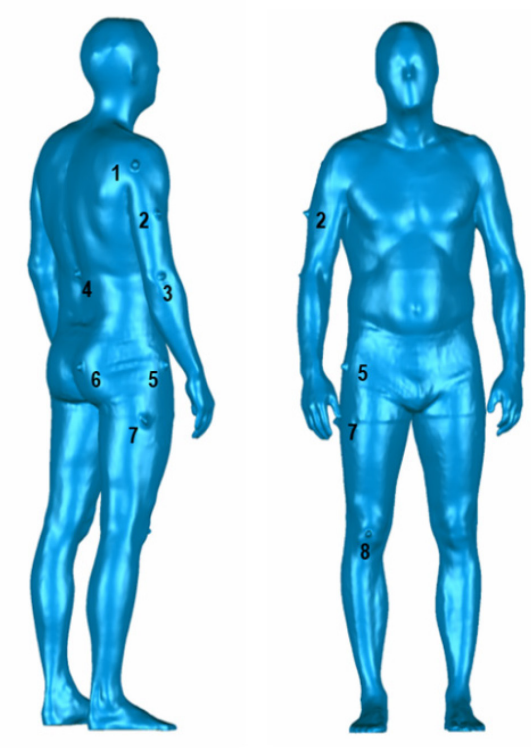

Fig. 2 Defined landmarks on standard position.

To enable valid measurement anthropometric landmarks were highlighted by polystyrene balls of $2 \mathrm{~cm}$ diameter. Fig. 2 shows the defined landmarks: 1. Mid armfold, 2. Position of largest girth upper arm, 3. Elbow, 4. Waist point at back, 5. Hip height at side, 6. Hip height at back, 7. Crotch height and 8. Mid of knee. Measurements were taken according to ISO 8559 standard: body height, chest girth, waist girth, hip girth, across back, upper arm length, upper arm girth, thigh girth, knee girth and calf girth. Yet, motion postures made adjustment necessary. For example, arm length was taken from mid armfold to wrist. This enables the identification of the maximal length. Leg length was taken in an adjusted method, too. It was 
measured not on the side but on the front of the leg, from crotch height over the knee and ankle to floor level.

The changes in scan surface were investigated by a three-step analysis: body measurements, cross sections and a 3D analysis. Therefore, the scans were segmented. The upper arm and the thigh area were cut at the joints (shoulder-elbow; hip-knee). The two upper arm segments and the three thigh segments were merged. The girths at the upper arm were measured from shoulder to elbow in stretched and flexed arm position (see Fig. 3). Girths were positioned in $1 \mathrm{~cm}$ distance to another. Depending on the upper arm lengths up to 21 measurements were taken. The differences in girth between stretched and flexed arms were investigated. The analysis showed differences between $-1.3 \mathrm{~cm}$ and $+2.8 \mathrm{~cm}$. The average deviation was $3.61 \%$. To illustrate the measurement differences due to movement the upper arm was segmented in three sections: close to shoulder, middle and close to elbow. The largest differences were identified in the section close to elbow.

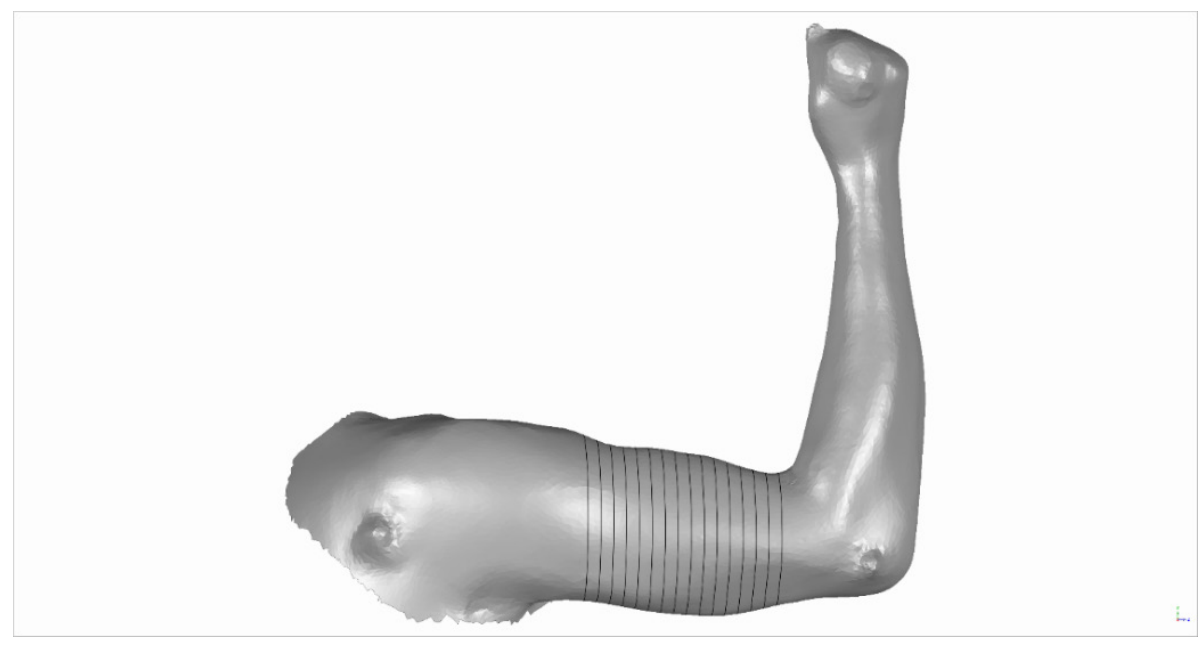

Fig. 3 Flexed arm with measurement positions.

The process to assess the thigh was similar to the upper arm analysis. Girths were measured from positions relaxed, leg flex and squat. For the leg flex movement it was assumed that the major muscle work would happen when the body weight is shifted to the foot on the platform. For the squat movement the end position was chosen. Starting from near crotch girths were positioned $1 \mathrm{~cm}$ distance from another. Depending on thigh lengths up to 33 measurements were taken. The analysis of the leg flex position showed differences between $-4.1 \mathrm{~cm}$ and $+1.8 \mathrm{~cm}$. The average deviation was $-2.7 \%$. The analysis of the squat position showed differences between $-9.5 \mathrm{~cm}$ and $+6.5 \mathrm{~cm}$. The average deviation was $0 \%$. The results from the test subjects varied extremely. To illustrate the measurement differences due to movement the thigh was segmented in three sections: close to hip, middle and close to knee. The largest differences were identified in the middle section in both assessed positions.

With the cross sections the middle of the upper arm and the thigh were assessed. The results indicate, that even when the girths show no significant value differences, the body form changes due to movement. Obviously width and depths of the body parts alter (see Fig. 4).

Finally, 3D analysis was performed to investigate the changes in geometry in regard of whole areas (upper arm and thigh). The generated heat maps show reductions in blue and increase in yellow/orange/red. Each color is correlated with a defined distance. The more intense the color the larger is the distance. Fig. 5 displays one result of a 3D analysis of the thigh in size 58 comparing the relaxed with leg flex position. From left to right the pictures show the front, side medial and the back view. There are reductions on the side and an increase of geometry in front and back. 
a

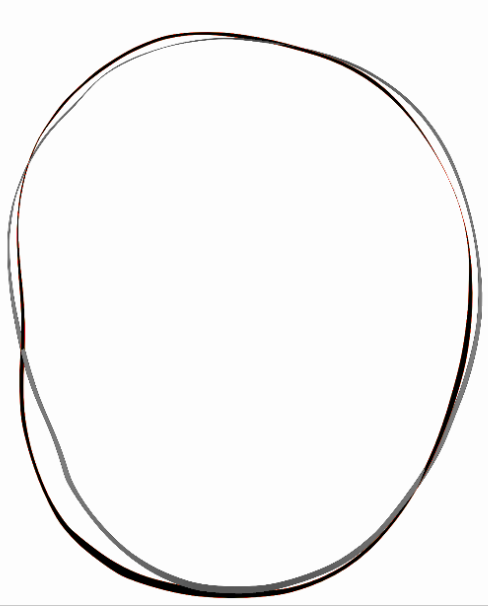

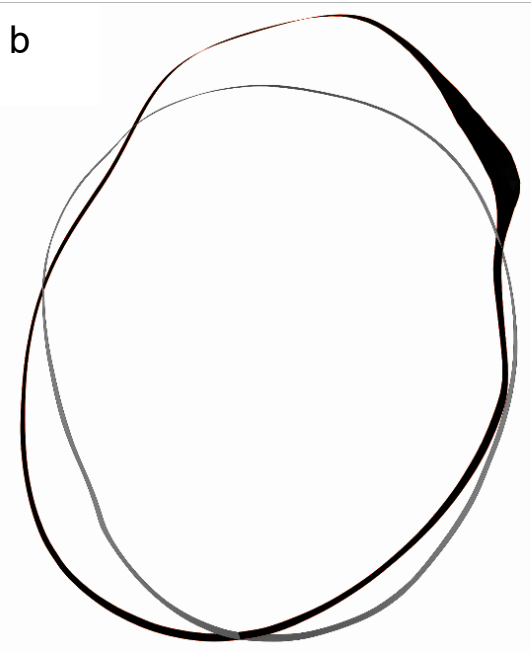

Fig. 4 (a) comparing cross section thigh relaxed (grey) with leg flex position (black); (b) comparing cross section thigh relaxed (grey) with squat position (black).
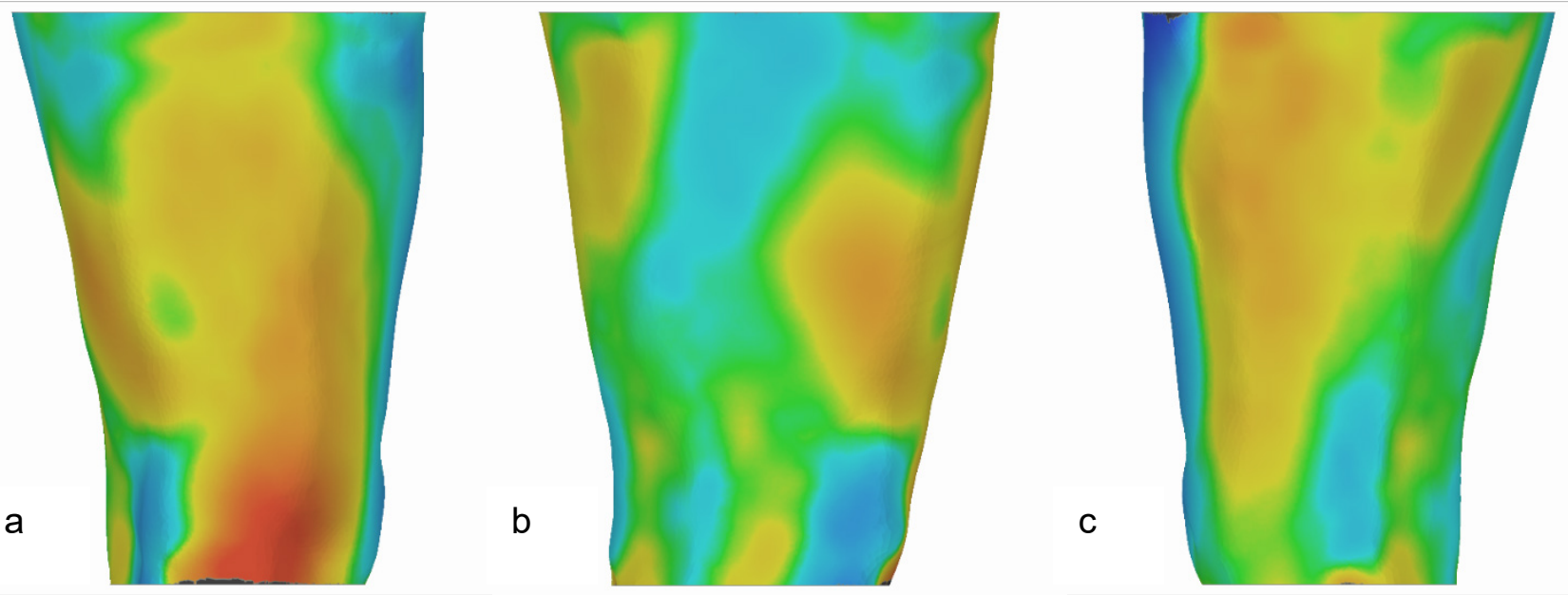

Fig. 5 Heat map comparison thigh relaxed with leg flex positon: (a) front view; (b) side view medial; (c) back view.

\section{Conclusion}

Garment fit in movement is a crucial for work and sportswear. Therefore, comprehensive dynamic anthropometric data is needed to develop garment with optimal ergonomic fit. To investigate the operational capability focusing clothing technology applications 3D photogrammetry full body scanner "Little Alice" from 3Dcopysystems was utilized to capture movements. A basic research was performed. Six parameters were examined by iterative tests: Shadowing of body areas, maximum of frames to be processed, acquisition area, movement speed, color and pattern of captured objects, data export and size accuracy. The results showed that 3D scan data shows appropriate accuracy to be used to analyze body measurements and geometry changes. Since, the low cost setup with 38 cameras represents the minimum amount to generate scans, limitations in quality were investigated. There was shadowing between arms and torso as well as between legs. Yet, compared to laser scanner systems there was less interference especially in movement. The acquisition area is rather small. Therefore, the range of motion must be considered when movements are defined. Though, three meaningful work and sports related movements were defined to assess body geometry alteration. It was found, that the dumbbell with $1 \mathrm{~kg}$ was not enough weight to lead to major muscle changes. Therefore, further tests with a larger workload should be performed. This is recommended with special view on the bigger sizes where the muscle form is less obvious due to the subcutaneous fat. The girth alteration at the upper arm in the section close to elbow were unexpected. These results need to be transferred in pattern development and further analyzed. The investigation of changes at thigh area were more difficult. There was shadowing between the legs 
especially in squat position. Here, the limitations of the system are more than obvious. Depending on the application it needs to be evaluated if an increase of cameras is reasonable. In addition, it seems of importance to enlarge the test sample and compare different target groups e.g. sporty and non-sporty as well as further sizes.

\section{Acknowledgements}

The IGF project $20121 \mathrm{~N}$ by the research association Forschungskuratorium Textil e.V., Reinhardtstraße 12-14, 10117 Berlin, was sponsored via the AIF as part of the program to support "Industrial Community Research and Development" (IGF), with funds from the Federal Ministry of Economics and Energy (BMWi) following an Order by the German Federal Parliament.

\section{References}

[1] Boorady, M.; Rucker, M.; Haise, C.; Ashdown, S. P. Protective Clothing for Pesticide Applicators: A Multimethod Needs Assessment, Journal of Textile And Apparel, Technology and Management, 2009, 6 (2), pp. 1-17.

[2] Bye, E.; Labat, K. L.; Delong, M. R. Analysis of Body Measurement Systems for Apparel, Clothing and Textiles Research Journal, 2006, 24 (2), pp. 66-79.

[3] Boorady, L. M. Functional clothing - Principles of fit, Indian Journal of Fibre \& Textile Research, 2011, 36, pp.344347.

[4] Schmid, U.; Mecheels, J. Kräfte an Textilien und Nähten der Kleidung in Abhängigkeit von Körperbewegungen und Kleidungsschnitt, Bekleidung \& Wäsche, 1981, 2, 77-82.

[5] Choi, S.; Ashdown, S. P. 3D body scan analysis of dimensional change in lower body measurements for active body positions, Textile Research Journal, 2011, 81(1), pp. 81-93.

[6] Temporal-3dMD Systems (4D). Available online: http://www.3dmd.com/static-3dmd_systems/dynamic-surfacemotion-capture-4d/ (accessed on 21.04.2020).

[7] 3D body shape movement. Available online: https://antropometria.ibv.org/en/laboratory/ (accessed on 21.04.2020).

[8] 4D Dynamic Scanner. Available online: https://ps.is.tuebingen.mpg.de/pages/4d-capture (accessed on 21.04.2020).

[9] Ashdown, S. P. Improving body movement comfort in apparel. In Improving comfort in clothing, Song, G., Woodhead Publishing, Cambridge, Great Britain, 2011, pp. 278-302.

[10] Morlock, S.; Lörcher, C.; Schenk, A. Entwicklung eines ergonomisch- und bewegungsorientierten Größensystems für Funktionsmaße zur optimierten Gestaltung von Berufs- und Schutzbekleidung / IGF 18993N, Hohenstein Institut fuer Textilinnovation gGmbH, Boennigheim, Germany, 2018.

[11] Bougourd, J. P.; Dekker, L. ; Ross, P. G.; Ward, J. P. A Comparison of Women's Sizing by 3D Electronic Scanning and Traditional Anthropometry, The Journal of The Textile Institute, 2000, 91(2), pp. 163-173.

[12] Brunsman, M. A.; Daanen, H. M.; Robinette, K. M. Optimal postures and positioning for human body scanning, Proceedings of the International Conference on Recent Advances in 3-D Digital Imaging and Modeling Ottawa, Ontario, Canada, 1997.

[13] Choi, S.-Y.; Ashdown, S. P. Application of Lower Body Girth Change Analysis Using 3D Body Scanning to Pants Patterns, Journal of the Korean Society of Clothing and Textiles, 2010, 34(6), pp. 955-968.

[14] Lee, J.; Ashdown, S. P. Upper Body Surface Change Analysis using 3-D Body Scanner, Journal of Korean Society of Clothing and Textiles, 2005, 29(12), pp. 1595-1607.

[15] Baytar, F.; Aultman, J.; Han, J. 3D Body Scanning for Examining Active Body Positions: An Exploratory Study for Re-Designing Scrubs, Proceedings of the 3rd International Conference on 3D Body Scanning Technologies, Lugano, Swiss, 2012.

[16] Nam, J.; Barnson, D. H.; Asdown, S. P. et al. Fit Analysis of Liquid Cooled Vest Prototypes Using 3D Body Scanning Technology, Journal of Textile and Apparel, Technology and Management, 2005, 4(3), pp. 1-15.

[17] Nawaz, N.; Troynikov, O.; Kennedy, K. Investigation into Fit, Distribution and Size of Air Gaps in Fire-Fighter Jackets to Female Body Form, Proceedings of the 3rd International Conference on 3D Body Scanning Technologies, Lugano, Swiss, 2012.

[18] Morlock, S.; Schenk, A. et al. 3D-basierte Entwicklung eines innovativen Verfahrens zur Passformdiagnose von Bekleidung / IGF 17763N, Hohenstein Institut für Textilinnovation gGmbH, Boennigheim, 2016.

[19] Choi, J.; Hong, K. 3D skin length deformation of lower body during knee joint flexion for the practical application of functional sportswear, Applied Ergonomics, 2015, 48, pp. 186-201.

[20] Gupta, D.; Zakaria, N. Anthropometry, apparel sizing and design, Woodhead Publishing Cambridge, Great Britain, 2014.

[21] Ebert, C. Neuentwickelter Stretchindex, Sport+Mode, 2015, 2, pp. 63.

[22] Park, S. I.; Hodgins, J. K. Capturing and Animating Skin Deformation in Human Motio, Proceedings of the SIGGRAPH 2006, Bosten, USA, 2006, pp. 881-889. 
[23] Aguiar, E. Performance Capture Methods, Proceedings of the European Conference on Computer Vision (ECCV), Zürich, Swiss, 2014.

[24] Wu, C.; Varanasi, K.; Liu, Y. et al. Shading-based Dynamic Shape Refinement from Multi-view Video under General Illumination, Proceedings of the IEEE International Conference on Computer Vision. Barcelona, Spain, 2011.

[25] Rose, B.; Murphy, M. Mapping wearer mobility for clothing design, European Publication Server, Cambridge, Great Britain, 2015.

[26] Fit to Deformation. Available online: http://www.drematrix.de/projects/fit-to-deformation/ (accessed on 23.04.2020). 